\title{
LINEARLY PRESENTED MODULES AND BOUNDS ON THE CASTELNUOVO-MUMFORD REGULARITY OF IDEALS
}

\author{
GIULIO CAVIGLIA AND ALESSANDRO DE STEFANI
}

\begin{abstract}
We estimate the Castelnuovo-Mumford regularity of ideals in a polynomial ring over a field by studying the regularity of certain modules generated in degree zero and with linear relations. In dimension one, this process gives a new type of upper bounds. By means of recursive techniques this also produces new upper bounds for ideals in any dimension.
\end{abstract}

\section{INTRODUCTION}

Let $S=\mathbb{k}\left[x_{1}, \ldots, x_{n}\right]$ be standard graded polynomial ring over a field $\mathbb{k}$, and $I$ be a homogeneous ideal generated in degree at most $D$. The Castelnuovo-Mumford regularity is a measure of the complexity of the ideal $I$, and has been extensively studied in the literature.

Upper bounds for $\operatorname{reg}(I)$ in terms of $D$ and $n$ have been proved by Galligo [Gal74, Gal79] and Giusti [Giu84] over fields of characteristic zero, then extended to all characteristics by Bayer and Mumford [BM93] and by the first named author and Sbarra [CS05]. They prove that $\operatorname{reg}(I) \leqslant(2 D)^{2^{n-2}}$.

Similar type of results for finitely generated graded modules, or for certain classes of modules or ideals, have been obtained by several authors (for instance, see [CFN08, BG09, HH06]).

A key result of this article is a new type of upper bound for modules of dimension one. This result is inspired by an analogous one obtained by Bruns, Conca and Römer in the zerodimensional case [BCR11, Remark 3.10 (b)].

For higher dimension, there is a well-known argument that allows to reduce to a lowerdimensional case by expressing the regularity of an ideal in terms of that of its hyperplane sections (see [CS05], and Section 2). We optimize this reduction step by introducing correction factors, therefore improving known upper bounds for the regularity of ideals in any dimension. The following is the main result of this article.

Theorem A. (Theorems 2.1 and 2.10) Let $\mathbb{k}$ be an infinite field, $S=\mathbb{k}\left[x_{1}, \ldots, x_{n}\right]$ with the standard grading, and $I$ be a homogeneous ideal generated in degree at most $D$. Let $d=\operatorname{dim}(S / I), h=\operatorname{ht}(I)$, and $y_{1}, \ldots, y_{d}$ be a linear system of parameters in $S / I$. Let e $(S / I)$ be the multiplicity of $S / I$, and $c$ be the value of Hilbert function of $S /\left(I+\left(y_{1}, \ldots, y_{d-1}\right)\right)$ in degree $D$ (if $d \leqslant 1$, by this we simply mean the value of the Hilbert function of $S / I$ in degree $D)$. Then

$$
\operatorname{reg}(S / I) \leqslant \begin{cases}D+c-1 & \text { if } d \leqslant 1 \\ {\left[(D+c-1)\left(D^{h}-\mathrm{e}(S / I)+1\right)\right]^{d^{d-2}}} & \text { if } d \geqslant 2 .\end{cases}
$$

2020 Mathematics Subject Classification. Primary 13D02; Secondary 13A02, 13 A15.

Key words and phrases. Linearly presented syzygies, Castelnuovo-Mumford regularity, EGH conjecture.

The work of the first named author was partially supported by a grant from the Simons Foundation (41000748, G.C.). 
For zero-dimensional ideals, a sharp upper bound is predicted by the Eisenbud-Green-Harris conjecture [EGH93, EGH96]. In Corollary 2.4 we compute this upper bound explicitly, and thanks to Theorem A we show show that it holds in some cases (see Remarks 2.6 and 2.7).

We would like to point out that, for general upper bounds on $\operatorname{reg}(S / I)$ such as the ones of Theorem A, a double exponential behavior in $n$ with base depending on $D$, is inevitable. This is because of a series of well-known examples due to Mayr and Meyer [MM82] (see also [BS88, Koh98]). However, thanks to Theorem A, we are able to drastically improve the upper bound $\operatorname{reg}(I) \leqslant(2 D)^{2^{n-2}}$ by dropping a factor of $2^{2^{n-2}}$. In fact, we prove that, if $n \geqslant 3$ and $I$ is a homogeneous ideal generated in degree at most $D$, then $\operatorname{reg}(I) \leqslant D^{2^{n-2}}$ (see Corollary 2.13).

\section{NEW UPPER BOUNDS ON THE REGULARITY OF IDEALS}

Throughout this article, $S$ denotes a standard graded polynomial ring $\mathbb{k}\left[x_{1}, \ldots, x_{n}\right]$ over a field $\mathbb{k}$, and $\mathfrak{m}=\left(x_{1}, \ldots, x_{n}\right)$ its graded maximal ideal. Replacing $\mathbb{k}$ with a field extension will not affect our considerations, therefore we will harmlessly assume throughout that $\mathbb{k}$ is infinite. Given a finitely generated graded $S$-module $M=\bigoplus_{j \in \mathbb{Z}} M_{j}$, we let $\operatorname{HF}(M ; j)=\operatorname{dim}_{\mathbb{k}}\left(M_{j}\right)$ denote its Hilbert function in degree $j \in \mathbb{Z}$. We let $\operatorname{reg}(M)=\sup \left\{i+j \mid H_{\mathfrak{m}}^{i}(M)_{j} \neq 0\right\}$ be its Castelnuovo-Mumford regularity, where $H_{\mathfrak{m}}^{i}(-)$ denotes the $i$-th graded local cohomology module with support in $\mathfrak{m}=\left(x_{1}, \ldots, x_{n}\right)$. Equivalently, $\operatorname{reg}(M)=\sup \left\{j-i \mid \operatorname{Tor}_{i}^{S}(\mathbb{k}, M)_{j} \neq 0\right\}$ (see [EG84]).

The next result, even if it involves rather basic techniques, is the crucial point of this article. We estimate the regularity of an ideal by means of the regularity of a related module, which turns out to be linearly presented. This fact, combined with a result of Chardin, Fall and Nagel [CFN08], leads to surprising linear upper bounds for the regularity of ideals of dimension at most one.

Theorem 2.1. Let $I$ be a homogeneous ideal such that $\operatorname{dim}(S / I) \leqslant 1$. Assume that $I$ is generated in degree at most $D$, and let $c=\operatorname{HF}(S / I ; D)$. Then $\operatorname{reg}(S / I) \leqslant D+c-1$.

Proof. If $c=0$, then there is nothing to prove. Moreover, when $n<2$ the claim is straightforward to check, so we will assume that $n \geqslant 2$. Let $s_{1}, \ldots, s_{c}$ be elements of $S$ whose images modulo $I$ form a $\mathbb{k}$-basis of $(S / I)_{D}$. Since $I+\left(s_{1}, \ldots, s_{c}\right) \subseteq \mathfrak{m}^{D}$, we have an exact sequence

$$
0 \longrightarrow U(-D) \longrightarrow S^{\oplus c}(-D) \stackrel{\left[s_{1}, \ldots, s_{c}\right]}{\longrightarrow} S / I \longrightarrow S / \mathfrak{m}^{D} \longrightarrow 0
$$

for some graded submodule $U$ of $F=S^{\oplus c}$. Thus, we obtain that $\operatorname{reg}(S / I) \leqslant \max \{D+$ $\left.\operatorname{reg}(F / U), \operatorname{reg}\left(S / \mathfrak{m}^{D}\right)\right\}=D+\operatorname{reg}(F / U)$.

From the exact sequences $\operatorname{Tor}_{2}^{S}\left(\mathbb{k}, S / \mathfrak{m}^{D}\right)_{j} \rightarrow \operatorname{Tor}_{1}^{S}(\mathbb{k}, F / U(-D))_{j} \rightarrow \operatorname{Tor}_{1}^{S}(\mathbb{k}, S / I)_{j}$ of vector spaces, we deduce that $U$ is generated in degree at most one, since both modules on the sides are zero for $j>D+1$. As a consequence, $F / U$ is a module of dimension at most one, generated by $c$ elements of degree zero and related in degrees at most one. If follows from [CFN08, Theorem 2.1 (ii)] applied to the case $l=1$ that $\operatorname{reg}(F / U) \leqslant c-1$, and the proof is complete.

For zero-dimensional ideals, the upper bound of Theorem 2.1 had already been proved by Bruns, Conca and Römer [BCR11, Remark 3.10 (b)]. However, we point out that in the zerodimensional case the best possible upper bound in terms of $D$ and $c$ is given by the EisenbudGreen-Harris Conjecture (for instance, see [EGH93, CM08]). In order to be more specific, we first recall some definitions and notation. Let $d_{1} \leqslant \ldots \leqslant d_{h}$ be non negative integers. An ideal $\mathcal{L}$ is called a $\left(d_{1}, \ldots, d_{h}\right)$-LPP ideal if $\mathcal{L}=\left(x_{1}^{d_{1}}, \ldots, x_{h}^{d_{h}}\right)+L$, where $L$ is a lex-segment ideal. 
We will make $L$ unique by always choosing the largest possible lex-segment ideal for which the equality holds. The Eisenbud-Green-Harris Conjecture (henceforth, EGH) states that, given any homogeneous ideal $I$ containing a regular sequence of degrees $d_{1}, \ldots, d_{h}$, there exists a $\left(d_{1}, \ldots, d_{h}\right)$-LPP ideal with the same Hilbert function as $I$.

Given a homogeneous ideal $I$ that contains a regular sequence of degrees $d_{1}, \ldots, d_{h}$, for $D \geqslant 0$ we let $\operatorname{LPP}\left(I ; d_{1}, \ldots, d_{h} ; D\right)$ be the $\left(d_{1}, \ldots, d_{h}\right)$-LPP ideal $\mathcal{L}=\left(x_{1}^{d_{1}}, \ldots, x_{h}^{d_{h}}\right)+L$ such that $L$ is either zero or is generated in degree $D$, and $\operatorname{HF}(S / I ; D)=\operatorname{HF}(S / \mathcal{L} ; D)$. An equivalent way of stating the EGH Conjecture is to assert that, for any homogeneous ideal $I$ containing a regular sequence of degrees $d_{1}, \ldots, d_{h}$, and for any integer $D \geqslant 0$, one has that $\operatorname{HF}(S / I ; D+1) \leqslant$ $\operatorname{HF}\left(S / \operatorname{LPP}\left(I ; d_{1}, \ldots, d_{h} ; D\right) ; D+1\right)$.

In what follows, whenever $h=n$ and $d_{1}=\ldots=d_{n}=D$, we denote $\operatorname{LPP}\left(I ; d_{1}, \ldots, d_{n} ; D\right)$ simply by $\operatorname{LPP}(I ; D)$. We ask the following question.

Question 2.2. Let $S=\mathbb{k}\left[x_{1}, \ldots, x_{n}\right]$, and $I$ be a zero-dimensional homogeneous ideal generated in degree at most $D$. Is $\operatorname{reg}(S / I) \leqslant \operatorname{reg}(S / \operatorname{LPP}(I ; D))$ ?

If the EGH Conjecture were to be true, then the Hilbert function of $S / I$ would be point-wise bounded above by the Hilbert function of $S / \operatorname{LPP}(I ; D)$, and this would clearly give a positive answer to Question 2.2. In this sense, Question 2.2 can be seen as a weaker version of the EGH Conjecture for zero-dimensional ideals.

In order to be more specific about the upper bound predicted by Question 2.2, we explicitly compute the regularity of a zero-dimensional LPP ideal.

Lemma 2.3. Let $d_{1} \leqslant \ldots \leqslant d_{n}$ and $D \geqslant 2$ be integers, with $D \leqslant \sum_{i=1}^{n}\left(d_{i}-1\right)$. Let $\mathcal{L}=\left(x_{1}^{d_{1}}, \ldots, x_{n}^{d_{n}}\right)+L$ be a $\left(d_{1}, \ldots, d_{n} ; D\right)$-LPP ideal, and assume that $\mathcal{L} \neq\left(x_{1}^{d_{1}}, \ldots, x_{n}^{d_{n}}\right)$. Set $u=x_{a}^{t_{a}} \cdot x_{a+1}^{t_{a+1}} \cdots x_{b}^{t_{b}}$, with $t_{a} \neq 0$, be the smallest monomial with respect to the lex order which has degree $D$, and belongs to $L$. Then $\operatorname{reg}(S / \mathcal{L})=t_{a}-1+\sum_{i=a+1}^{n}\left(d_{i}-1\right)$.

Proof. Let $s=\sum_{i=1}^{n}\left(d_{i}-1\right)$ and $J=\left(x_{1}^{d_{1}}, \ldots, x_{n}^{d_{n}}\right)$. Let $j \leqslant s$, and let $v_{j}$ be the smallest monomial with respect to the lex order which has degree $j$, and does not belong to $J$. Then $v_{j} \in \mathcal{L}$ if and only if $S_{j} \subseteq \mathcal{L}_{j}$. Thus, the regularity of $S / \mathcal{L}$ is achieved in the highest degree $j$ for which $v_{j}$ does not belong to $\mathcal{L}$. It is easy to see that, if $v_{j}=x_{1}^{j_{1}} \cdots x_{n}^{j_{n}}$, and $i$ is the smallest index for which $j_{i} \neq 0$, then $j_{r}=d_{r}-1$ for all $r>i$, and $v_{j-1}=x_{i}^{j_{i}-1} x_{i+1}^{d_{i+1}-1} \cdots x_{n}^{d_{n}-1}$. Therefore, $v_{j} \notin \mathcal{L}$ if and only if $j_{r}=0$ for all $r \in\{1, \ldots, a-1\}$, and $j_{a}<t_{a}$. The largest $j$ for which $v_{j}$ satisfies this condition is $j=t_{a}-1+\sum_{i=a+1}^{n}\left(d_{i}-1\right)$.

The previous considerations, together with Lemma 2.3, lead to the following result.

Corollary 2.4. Let $I$ be a zero-dimensional ideal generated in degree at most $D$. If the EGH Conjecture holds true, then in the same notation as in Lemma 2.3 we have that

$$
\operatorname{reg}(S / I) \leqslant(n-a)(D-1)+t_{a}-1 .
$$

Remark 2.5. Assuming the validity of the EGH Conjecture, the upper bound of Corollary 2.4 is clearly sharp, as it is achieved by $S / \operatorname{LPP}(I ; D)$.

Remark 2.6. In the setup of Corollary 2.4, if we write $\operatorname{LPP}(I ; D)=\left(x_{1}^{D}, \ldots, x_{n}^{D}\right)+L$ and we assume that $c=\operatorname{HF}(S / I ; D)<D$, then the smallest monomial of degree $D$ inside $L$ is $x_{n-1}^{c+1} x_{n}^{D-(c+1)}$. It then follows from Lemma 2.3 that $\operatorname{reg}(S / \operatorname{LPP}(I ; D))=c+D-1$, which is the upper bound obtained in Theorem 2.1. It follows that Question 2.2 has positive answer when $c<D$. 
If more information about the ideal $I$ is available, then one can aim at sharper upper bounds. For instance, if $I$ contains a regular sequence of degrees $d_{1} \leqslant \ldots \leqslant d_{n}$, and the EGH Conjecture with respect to this sequence of degrees holds true, then $\operatorname{reg}(S / I) \leqslant \operatorname{reg}\left(S / \operatorname{LPP}\left(I ; d_{1}, \ldots, d_{n} ; D\right)\right)$ for all $D \geqslant 0$. Note that an explicit formula for the right-hand side can be obtained by means of Lemma 2.3. One concrete example in which this remark applies is when $d_{i+1} \geqslant \sum_{j=1}^{i}\left(d_{i}-1\right)$ for all $i=1, \ldots, n-1$, as proved in [CDS20, Theorem A].

Remark 2.7. In the setup of Corollary 2.4, if $d_{1} \leqslant \ldots \leqslant d_{n} \leqslant D$, then one can show that $\operatorname{HF}(\operatorname{LPP}(I ; D) ; j) \leqslant \operatorname{HF}\left(\operatorname{LPP}\left(I ; d_{1}, \ldots, d_{n} ; D\right) ; j\right)$ for all $j \in \mathbb{Z}$. Therefore, if the EGH is known to hold for a sequence of degrees $d_{1} \leqslant \ldots \leqslant d_{n} \leqslant D$, then Question 2.2 has a positive answer for ideals that contain a regular sequence of degrees $d_{1}, \ldots, d_{n}$.

We now return to our original goal of producing improved upper bounds for the CastelnuovoMumford regularity of ideals in any dimension. More specifically, we aim at improving a standard recursive argument used in [CS05], which allows to drop the dimension by one at a time, by including several correction terms. Once we reach dimension one, we will finally use the new bounds obtained in Theorem 2.1. Let us introduce some notation first.

Let $I$ be a homogeneous ideal, and set $R=S / I$. Let $y_{1}, \ldots, y_{t}$ be a sequence of linear forms in $R$, and for $i=1, \ldots, t$ let $R^{(i)}$ denote the $\operatorname{ring} R /\left(y_{1}, \ldots, y_{i}\right)$. For convenience, we let $R^{(0)}=R$. We recall that a homogeneous non-zero element $y$ is called filter regular for $R$ if $0:_{R} y$ has finite length. The sequence $y_{1}, \ldots, y_{t}$ is called filter regular for $R$ if $y_{i+1}$ is filter regular over $R^{(i)}$ for all $i \in\{0, \ldots, t-1\}$. If $\mathbb{k}$ is infinite, a sufficiently general linear form $\ell \in R^{(i)}$ is a filter regular element for $R^{(i)}$. In particular, a sufficiently general choice of minimal generators of $\mathfrak{m}$ forms a filter regular sequence for $R$. Let $y_{1}, \ldots, y_{t}$ be a filter regular sequence for $R$. Observe that, in this case, $\left(I+\left(y_{1}, \ldots, y_{i}\right)\right)^{\text {sat }}=\left(I+\left(y_{1}, \ldots, y_{i}\right)\right): \mathfrak{m}^{\infty}=\left(I+\left(y_{1}, \ldots, y_{i}\right)\right): y_{i+1}^{\infty}$ for all $0 \leqslant i \leqslant t-1$, where for $i=0$ we simply mean that $I^{\text {sat }}=I: y_{1}^{\infty}$.

Now assume that $R=S / I$ has Krull dimension $d>1$, and $I$ is generated in degree at most $D$. Let $y_{1}, \ldots, y_{d}$ be a filter regular sequence for $R$ consisting of linear forms. If $\ell(-)$ denotes the length of a module, it is shown in the proof of [CS05, Theorem 2.4] that

$$
\operatorname{reg}(R) \leqslant \max \left\{D, \operatorname{reg}\left(R^{(1)}\right)\right\}+\left(\prod_{i=1}^{d-1} \operatorname{reg}\left(R^{(i)}\right)\right) \cdot \ell\left(\frac{\left(I+\left(y_{1}, \ldots, y_{d-1}\right)\right)^{\mathrm{sat}}+\left(y_{d}\right)}{I+\left(y_{1}, \ldots, y_{d}\right)}\right)
$$

Observe that

$$
\begin{aligned}
\ell\left(\frac{\left(I+\left(y_{1}, \ldots, y_{d-1}\right)\right)^{\mathrm{sat}}+\left(y_{d}\right)}{I+\left(y_{1}, \ldots, y_{d}\right)}\right) & =\ell\left(R^{(d)}\right)-\ell\left(\frac{S}{\left(I+\left(y_{1}, \ldots, y_{d-1}\right)\right)^{\mathrm{sat}}+\left(y_{d}\right)}\right) \\
& =\ell\left(R^{(d)}\right)-\mathrm{e}(R) .
\end{aligned}
$$

A typical estimate for $\ell\left(R^{(d)}\right)$ comes from the fact that we can find forms $f_{1}, \ldots, f_{h} \in I$ of degree $D$ such that $f_{1}, \ldots, f_{h}, y_{1}, \ldots, y_{d}$ forms a regular sequence of maximal length in $S$. Thus $\ell\left(R^{(d)}\right) \leqslant \ell\left(S /\left(f_{1}, \ldots, f_{h}, y_{1}, \ldots, y_{d}\right)\right)=D^{h}$. We here provide a slightly more refined estimate for $\ell\left(R^{(d)}\right)$.

To avoid any confusion, and to be consistent with the notation used in the proof of Theorem 2.10 , in the next lemma we consider a polynomial ring in $h$ variables, rather than in $n$ variables. 
Lemma 2.8. Let $S=\mathbb{k}\left[x_{1}, \ldots, x_{h}\right]$, and $I \subseteq S$ be a homogeneous ideal, generated in degree at most $D$, and such that $R=S / I$ is Artinian. Let $c^{\prime}=\operatorname{HF}(R ; D)$. Then

$$
\ell(R) \leqslant D^{h}-\left(\begin{array}{c}
D+h-1 \\
h-1
\end{array}\right)+c^{\prime}+h \leqslant D^{h} .
$$

Proof. Since $R$ is Artinian and generated in degree at most $D$ the ideal $I$ contains an ideal $J$ generated a regular sequence of $h$ forms of degree $D$. Therefore, we get

$$
\begin{aligned}
\ell(R) & =\ell(S / J)-\ell(I / J) \leqslant D^{h}-\operatorname{HF}(I / J ; D) \\
& =D^{h}-\operatorname{HF}(I ; D)+\operatorname{HF}(J ; D) \leqslant D^{h}-\left(\begin{array}{c}
D+h-1 \\
h-1
\end{array}\right)+c^{\prime}+h .
\end{aligned}
$$

Since $c^{\prime}=\operatorname{HF}(S ; D)-\operatorname{HF}(I ; D) \leqslant\left(\begin{array}{c}D+h-1 \\ h-1\end{array}\right)-h$ always holds, the proof is complete.

Notation 2.9. We let $\Phi\left(D, c^{\prime}, h\right)=D^{h}-\left(\begin{array}{c}D+h-1 \\ h-1\end{array}\right)+c^{\prime}+h$.

We are finally ready to state and prove the second part of our main result.

Theorem 2.10. Let $S=\mathbb{k}\left[x_{1}, \ldots, x_{n}\right]$, and $I \subseteq S$ be a homogeneous ideal of height $h \leqslant n-2$, generated in degree at most $D$, and let $d=n-h=\operatorname{dim}(S / I) \geqslant 2$. Let $y_{1}, \ldots, y_{d}$ be a system of parameters for $R=S / I$ consisting of linear forms. Let $c=\operatorname{HF}\left(R /\left(y_{1}, \ldots, y_{d-1}\right) ; D\right)$, and $c^{\prime}=\operatorname{HF}\left(R /\left(y_{1}, \ldots, y_{d}\right) ; D\right)$. Then

$$
\operatorname{reg}(R) \leqslant\left((D+c-1)\left(\Phi\left(D, c^{\prime}, h\right)-\mathrm{e}(R)+1\right)\right)^{2^{d-2}} .
$$

Proof. If $z_{1}, \ldots, z_{d}$ is a general choice of a linear system of parameters, then for all $t \in$ $\{1, \ldots, d\}$ and all $j \in \mathbb{Z}$ we have that $\operatorname{HF}\left(R /\left(z_{1}, \ldots, z_{t}\right) ; j\right) \leqslant \operatorname{HF}\left(R /\left(y_{1}, \ldots, y_{t}\right) ; j\right)$. Therefore, after possibly replacing $y_{1}, \ldots, y_{d}$ with a general choice of a linear system of parameters, we may assume $y_{1}, \ldots, y_{d}$ is a filter regular sequence for $R$. For $i=1, \ldots, d$, let $R^{(i)}=R /\left(y_{1}, \ldots, y_{i}\right)$. Applying $(2.1)$ to $R^{(1)}$ yields $\operatorname{reg}\left(R^{(1)}\right) \leqslant \max \left\{D, \operatorname{reg}\left(R^{(2)}\right)\right\}+A_{2}$, where we set $A_{2}=\operatorname{reg}\left(R^{(2)}\right) \cdots \operatorname{reg}\left(R^{(d-1)}\right) \cdot\left(\ell\left(R^{(d)}\right)-\mathrm{e}(R)\right)$. Applying (2.1) to $R$, and using the estimate for $\operatorname{reg}\left(R^{(1)}\right)$ which we have just obtained, we get that

$$
\begin{aligned}
\operatorname{reg}(R) & \leqslant \max \left\{D, \operatorname{reg}\left(R^{(2)}\right)\right\}+A_{2}+\left(\max \left\{D, \operatorname{reg}\left(R^{(2)}\right)\right\}+A_{2}\right) \cdot A_{2} \\
& =\left(1+A_{2}\right) \cdot\left(\max \left\{D, \operatorname{reg}\left(R^{(2)}\right)\right\}+A_{2}\right) \leqslant\left(\max \left\{D, \operatorname{reg}\left(R^{(2)}\right)\right\}+A_{2}\right)^{2} .
\end{aligned}
$$

Repeating the same argument for $R^{(j)}$, with $2 \leqslant j \leqslant d-2$, we obtain that

$$
\operatorname{reg}(R) \leqslant\left(\max \left\{D, \operatorname{reg}\left(R^{(d-1)}\right)\right\}+A_{d-1}\right)^{2^{d-2}} \leqslant\left((D+c-1)+A_{d-1}\right)^{2^{d-2}},
$$

where $A_{d-1}=\operatorname{reg}\left(R^{(d-1)}\right) \cdot\left(\ell\left(R^{(d)}\right)-\mathrm{e}(R)\right)$ and the last inequality follows from the fact that $\max \left\{D, \operatorname{reg}\left(R^{(d-1)}\right)\right\} \leqslant D+c-1$, by Theorem 2.1. By Lemma 2.8 we have $\ell\left(R^{(d)}\right) \leqslant \Phi\left(D, c^{\prime}, h\right)$, and thus $A_{d-1} \leqslant(D+c-1)\left(\Phi\left(D, c^{\prime}, h\right)-\mathrm{e}(R)\right)$ follows again from Theorem 2.1. We conclude that

$$
\operatorname{reg}(R) \leqslant\left((D+c-1)\left(\Phi\left(D, c^{\prime}, h\right)-\mathrm{e}(R)+1\right)\right)^{2^{d-2}} .
$$

Remark 2.11. By Lemma 2.8 we have that $\Phi\left(D, c^{\prime}, h\right) \leqslant D^{h}$, and therefore the bound

$$
\operatorname{reg}(R) \leqslant\left((D+c-1)\left(D^{h}-\mathrm{e}(R)+1\right)\right)^{2^{d-2}}
$$

claimed in Theorem A now follows from Theorem 2.10. 
Remark 2.12. Let $R=S / I$ be a $d$-dimensional ring and $a=\operatorname{HF}(R ; D)$. Let $y_{1}, \ldots, y_{d}$ be a linear system of parameters for $S / I$, let $c=\operatorname{HF}\left(R /\left(y_{1}, \ldots, y_{d-1}\right) ; D\right)$ and $c^{\prime}=\operatorname{HF}\left(R /\left(y_{1}, \ldots, y_{d}\right) ; D\right)$, as in Theorem 2.10. Consider the $D$-th Macaulay expansion of $a$ (for instance, see [BH93]):

$$
a=\left(\begin{array}{c}
a_{1} \\
D
\end{array}\right)+\left(\begin{array}{c}
a_{2} \\
D-1
\end{array}\right)+\cdots+\left(\begin{array}{c}
a_{D} \\
1
\end{array}\right)
$$

A repeated application of Green's hyperplane restriction Theorem [Gre89] yields

$$
c \leqslant\left(\begin{array}{c}
a_{1}-(d-1) \\
D
\end{array}\right)+\left(\begin{array}{c}
a_{2}-(d-1) \\
D-1
\end{array}\right)+\cdots+\left(\begin{array}{c}
a_{D}-(d-1) \\
1
\end{array}\right)
$$

and

$$
c^{\prime} \leqslant\left(\begin{array}{c}
a_{1}-d \\
D
\end{array}\right)+\left(\begin{array}{c}
a_{2}-d \\
D-1
\end{array}\right)+\cdots+\left(\begin{array}{c}
a_{D}-d \\
1
\end{array}\right)
$$

Since the right-hand side of the inequality in Theorem 2.10 is increasing in $c$ and $c^{\prime}$, one can replace their values with the above estimates, and still obtain an upper bound on $\operatorname{reg}(R)$. Alternatively, one can use the Macaulay expansion of $c$ to determine an upper bound for $c^{\prime}$. If $c=\left(\begin{array}{l}c_{1} \\ D\end{array}\right)+\left(\begin{array}{c}c_{2} \\ D-1\end{array}\right)+\cdots+\left(\begin{array}{c}c_{D} \\ 1\end{array}\right)$, then $c^{\prime} \leqslant\left(\begin{array}{c}c_{1}-1 \\ D\end{array}\right)+\left(\begin{array}{c}c_{2}-1 \\ D-1\end{array}\right)+\cdots+\left(\begin{array}{c}c_{D}-1 \\ 1\end{array}\right)$.

Theorem 2.10 allows to drastically improve the upper bound $\operatorname{reg}(I) \leqslant(2 D)^{2^{n-2}}$ which is usually found in the literature. In fact, it can be shown that $\operatorname{reg}(S / I) \leqslant D^{2^{n-2}}$ holds for all $n \geqslant 3$ as a consequence of Theorem 2.10.

Corollary 2.13. Let $S=\mathbb{k}\left[x_{1}, \ldots, x_{n}\right]$ with the standard grading, and $I$ be a homogeneous ideal, generated in degree at most $D$. Assume that $n \geqslant 3$. Then $\operatorname{reg}(I) \leqslant D^{2^{n-2}}$.

Proof. Let $d=\operatorname{dim}(S / I)$ and $h=n-d=\operatorname{ht}(I)$. We may assume that $D \geqslant 2$. If $h=1$, then we can write $I=f I^{\prime}$ for some $f \in S$ of degree $D^{\prime} \leqslant D$ and $I^{\prime}$ an ideal of height at least two, generated in degree at most $D-D^{\prime}$. Since $\operatorname{reg}(I)=D^{\prime}+\operatorname{reg}\left(I^{\prime}\right)$, the claim is clear provided we can prove the corollary for ideals of height at least two.

After a change of coordinates, we may assume that $x_{n}, \ldots, x_{h+1}$ forms a linear system of parameters which form a filter regular sequence for $R=S / I$. Let $\bar{S}=\mathbb{k}\left[x_{1}, \ldots, x_{h+1}\right]$, so that $R /\left(x_{n}, \ldots, x_{h+2}\right)$ can be viewed as a quotient of $\bar{S}$ by a homogeneous ideal $\bar{I}$, still generated in degree at most $D$.

If $\operatorname{dim}(R) \leqslant 1$, then $\operatorname{reg}(R) \leqslant(D-1) n$ by [CFN08, Theorem 3.5]. The latter is easily seen to be bounded above by $D^{2^{n-2}}-1$ whenever $n \geqslant 3$ and $D \geqslant 2$. Thus, $\operatorname{reg}(I)=\operatorname{reg}(R)+1 \leqslant D^{2^{n-2}}$.

For the rest of the proof, assume $\operatorname{dim}(R) \geqslant 2$. Observe that $c=\operatorname{HF}(\bar{S} / \bar{I} ; D) \leqslant \operatorname{HF}(\bar{S} ; D)-$ ht $(\bar{I})=\left(\begin{array}{c}D+h \\ h\end{array}\right)-h$ and, if we let $S^{\prime}=\bar{S} /\left(x_{h+1}\right)$ we similarly have that $c^{\prime} \leqslant \operatorname{HF}\left(S^{\prime} ; D\right)-$ $\operatorname{ht}\left(I S^{\prime} ; D\right)=\left(\begin{array}{c}D+h-1 \\ h-1\end{array}\right)-h$.

Observe that, in the above inequalities, equality holds for $c$ if and only if it holds for $c^{\prime}$, and this happens if and only if $I$ is generated by a regular sequence of forms of degree $D$. When this is the case we have $\operatorname{reg}(R)=(D-1) h$, which can be bounded above by $D^{2^{h-1}}-1$ for all $h \geqslant 2$, as a straightforward calculations shows. Since $h \leqslant n-2$, the claim follows in this case as well. 
We may henceforth assume that $c \leqslant\left(\begin{array}{c}D+h \\ h\end{array}\right)-h-1$ and $c^{\prime} \leqslant\left(\begin{array}{c}D+h-1 \\ h-1\end{array}\right)-h-1$. In this case, we have that $\Phi\left(D, c^{\prime}, h\right)=D^{h}-\left(\begin{array}{c}D+h-1 \\ h-1\end{array}\right)+c^{\prime}+h \leqslant D^{h}-1$. By Theorem 2.10 we get that

$$
\begin{aligned}
\operatorname{reg}(R) & \leqslant\left[(D+c-1)\left(\Phi\left(D, c^{\prime}, h\right)-\mathrm{e}(R)+1\right)\right]^{2^{n-h-2}} \\
& \leqslant\left[\left(D+\left(\begin{array}{c}
D+h \\
h
\end{array}\right)-h-2\right)\left(D^{h}-\mathrm{e}(R)\right)\right]^{2^{n-h-2}} .
\end{aligned}
$$

A straightforward computation shows that for all $D \geqslant 2$ and $h \geqslant 2$ one has $D+\left(\begin{array}{c}D+h \\ h\end{array}\right)-h-2 \leqslant$ $D^{h}$. Since $2 h \leqslant 2^{h}$ for all $h \geqslant 2$, and because $\mathrm{e}(R) \geqslant 1$, we finally get

$$
\operatorname{reg}(R) \leqslant\left[D^{h}\left(D^{h}-\mathrm{e}(R)\right)\right]^{2^{n-h-2}}<D^{2 h\left(2^{n-h-2}\right)} \leqslant D^{2^{n-2}},
$$

so that $\operatorname{reg}(I)=\operatorname{reg}(R)+1 \leqslant D^{2^{n-2}}$ in this case as well.

We point out that the bound of Corollary 2.13 also follows from [CFN08, Example 3.6]. However, increasing either $D$ or the height $h$ of the ideal $I$, and conducting a careful analysis of the quantities involved in the right-hand side of the inequality in Theorem 2.10, leads to even more refined estimates than the one above.

\section{REFERENCES}

[BCR11] Winfried Bruns, Aldo Conca, and Tim Römer. Koszul cycles. In Combinatorial aspects of commutative algebra and algebraic geometry, volume 6 of Abel Symp., pages 17-33. Springer, Berlin, 2011. 1, 2

[BG09] M. Brodmann and T. Götsch. Bounds for the Castelnuovo-Mumford regularity. J. Commut. Algebra, 1(2):197-225, 2009. 1

[BH93] Winfried Bruns and Jürgen Herzog. Cohen-Macaulay rings, volume 39 of Cambridge Studies in Advanced Mathematics. Cambridge University Press, Cambridge, 1993. 6

[BM93] Dave Bayer and David Mumford. What can be computed in algebraic geometry? In Computational algebraic geometry and commutative algebra (Cortona, 1991), Sympos. Math., XXXIV, pages 1-48. Cambridge Univ. Press, Cambridge, 1993. 1

[BS88] David Bayer and Michael Stillman. On the complexity of computing syzygies. volume 6, pages 135-147. 1988. Computational aspects of commutative algebra. 2

[CDS20] Giulio Caviglia and Alessandro De Stefani. The Eisenbud-Green-Harris conjecture for fast-growing degree sequences. arXiv: 2007.15467, 2020. 4

[CFN08] Marc Chardin, Amadou Lamine Fall, and Uwe Nagel. Bounds for the Castelnuovo-Mumford regularity of modules. Math. Z., 258(1):69-80, 2008. 1, 2, 6, 7

[CM08] Giulio Caviglia and Diane Maclagan. Some cases of the Eisenbud-Green-Harris conjecture. Math. Res. Lett., 15(3):427-433, 2008. 2

[CS05] Giulio Caviglia and Enrico Sbarra. Characteristic-free bounds for the Castelnuovo-Mumford regularity. Compos. Math., 141(6):1365-1373, 2005. 1, 4

[EG84] David Eisenbud and Shiro Goto. Linear free resolutions and minimal multiplicity. J. Algebra, 88(1):89133, 1984. 2

[EGH93] David Eisenbud, Mark Green, and Joe Harris. Higher Castelnuovo theory. Number 218, pages 187-202. 1993. Journées de Géométrie Algébrique d'Orsay (Orsay, 1992). 2

[EGH96] David Eisenbud, Mark Green, and Joe Harris. Cayley-Bacharach theorems and conjectures. Bull. Amer. Math. Soc. (N.S.), 33(3):295-324, 1996. 2

[Gal74] André Galligo. à propos du théorème de-préparation de Weierstrass. In Fonctions de plusieurs variables complexes (Sém. François Norguet, octobre 1970-décembre 1973; à la mémoire d'André Martineau), pages 543-579. Lecture Notes in Math., Vol. 409. 1974. Thèse de 3ème cycle soutenue le 16 mai 1973 à l'Institut de Mathématique et Sciences Physiques de l'Université de Nice. 1

[Gal79] André Galligo. Théorème de division et stabilité en géométrie analytique locale. Ann. Inst. Fourier (Grenoble), 29(2):vii, 107-184, 1979. 1 
[Giu84] M. Giusti. Some effectivity problems in polynomial ideal theory. In EUROSAM 84 (Cambridge, 1984), volume 174 of Lecture Notes in Comput. Sci., pages 159-171. Springer, Berlin, 1984. 1

[Gre89] Mark Green. Restrictions of linear series to hyperplanes, and some results of Macaulay and Gotzmann. In Algebraic curves and projective geometry (Trento, 1988), volume 1389 of Lecture Notes in Math., pages 76-86. Springer, Berlin, 1989. 6

[HH06] Lê Tuân Hoa and Eero Hyry. Castelnuovo-Mumford regularity of canonical and deficiency modules. J. Algebra, 305(2):877-900, 2006. 1

[Koh98] Jee Koh. Ideals generated by quadrics exhibiting double exponential degrees. J. Algebra, 200(1):225245, 1998. 2

[MM82] Ernst W. Mayr and Albert R. Meyer. The complexity of the word problems for commutative semigroups and polynomial ideals. Adv. in Math., 46(3):305-329, 1982. 2

Department of Mathematics, Purdue University, 150 N. University Street, West Lafayette, IN 47907-2067, USA

Email address: gcavigli@purdue.edu

Dipartimento di Matematica, Università di Genova, Via Dodecaneso 35, 16146 Genova, Italy

Email address: destefani@dima.unige.it 